\title{
Síndrome da dilatação intestinal suína
}

\author{
Porcine intestinal distension syndrome
}

Eliana Silva Paladino ${ }^{I}$ Roberto Maurício Carvalho Guedes ${ }^{\text {** }}$

\section{- REVISÃO BIBLIOGRÁFICA -}

\section{RESUMO}

A síndrome da dilatação intestinal suína (PIDS) é causa importante de morte súbita em suínos em fase de crescimento e terminação. Acomete animais aparentemente saudáveis, em boa conformação corporal e sem histórico prévio de doença gastrointestinal, cursa com intensa dilatação abdominal aguda, de evolução rápida, levando à dispnéia e morte por asfixia em até duas horas. A patogênese não está plenamente esclarecida, porém há hipóteses sobre fatores de risco associados ao manejo, aos hábitos alimentares e tipo de alimentação fornecida aos suínos. São discutidos nesta revisão os principais fatores predisponentes para a ocorrência de PIDS, sua fisiopatologia e o diagnóstico adequado dessa afecção. As causas de morte súbita devem ser devidamente avaliadas e os fatores predisponentes à PIDS devem ser determinados para melhor efetividade de diagnóstico e controle de mortalidade.

Palavras-chave: dilatação intestinal, morte súbita, síndrome hemorrágica suína, suínos, torção intestinal.

\section{ABSTRACT}

The porcine intestinal distension syndrome (PIDS) is a major cause of sudden death in growing and finishing pigs. It affects apparently healthy animals in good body conformation and without prior evidence of gastrointestinal disease. PIDS presents as an acute intense abdominal distension with rapid evolution, leading to respiratory distress and death by asphyxiation within two hours. The pathogenesis is not fully understood, but there are a few hypotheses about risk factors associated with management, feed intake and type of feed offered to the pigs. In this review, the main predisposing factors for the occurrence of PIDS, its physiopathology and the proper diagnosis of this condition are discussed. Causes of sudden death must be properly assessed and risk factors must be determined for better effectiveness of diagnosis and mortality control.

Key words: intestinal distension, sudden death, porcine hemorrhagic bowel syndrome, swine, intestinal torsion.

\section{INTRODUÇÃO}

A morte súbita de suínos é um evento comum dentro de sistemas de produção e a causa normalmente não é diagnosticada. Com frequência, existe envolvimento do sistema digestivo nesses quadros e diversos são os diagnósticos diferenciais, como torção gástrica, volvo e úlceras gastroesofágicas (SMITH \& SHANKS, 1971; ALLEN \& SAUNDERS, 1976; BUDDLE \& TWOMEY, 2002; THOMSON et al., 2007). Morte súbita é classificada como acidental, imprevisível e inevitável, porém vem sendo relatada como afecção comum com impacto na produção (STRAW et al., 2002; HOLLIS, 2006; MARTINEAU, 2008a).

A síndrome da dilatação intestinal suína (porcine intestinal distension syndrome - PIDS) é a terminologia utilizada para se referir a um quadro de morte súbita de leitões em fase de crescimento e

'Departamento de Clínica e Cirurgia Veterinárias, Escola de Veterinária, Universidade Federal de Minas Gerais (UFMG). Av. Antônio Carlos, 6627, 31270-901, Pampulha, Belo Horizonte, MG, Brasil. E-mail: guedesufmg@ gmail.com. *Autor para correspondência. 
terminação, de evolução rápida, caracterizada na avaliação post mortem por cadáveres acentuadamente pálidos e com intensa dilatação abdominal, em animais que não apresentavam sinais clínicos de doença prévia. Por não ter uma etiologia bem definida, é comum que se encontre outras denominações, como síndrome hemorrágica intestinal (Hemorrhagic Bowel Syndrome - HBS) (SMITH \& SHANKS, 1971; ALLEN \& SAUNDERS, 1976; BUDDLE \& TWOMEY, 2002; STRAW et al., 2002; HOLLIS, 2006; THOMSON et al., 2007; MARTINEAU, 2008a; MARTINEAU et al., 2008; LABUSCAGNE, 2009), intestino vermelho (Red Gut) (SMITH \& SHANKS, 1971; LABUSCAGNE, 2009), timpanismo por soro de leite (whey bloat), torção de mesentério, entre outras (BUDDLE \& TWOMEY, 2002; STRAW et al., 2002; THOMSON et al., 2007; MARTINEAU, 2008a; MARTINEAU et al., 2008).

A ocorrência é esporádica e varia entre propriedades. Em uma mesma granja, existe variação entre lotes (HOLLIS, 2006; MARTINEAU, 2008a). Há relatos, desde a década de 1970, de ocorrência em todo o mundo, incluindo Escócia (SMITH \& SHANKS, 1971), Estados Unidos (STRAW et al., 2002; HOLLIS, 2006), Canadá (STRAW et al., 2002), Reino Unido (ALLEN \& SAUNDERS, 1976; BUDDLE \& TWOMEY, 2002), Áustria, Suíça, Noruega, Austrália, Nova Zelândia (BUDDLE \& TWOMEY, 2002), África do Sul (LABUSCAGNE, 2009) e Brasil (DESROSIERS, 2008), porém a falta de diagnósticos precisos que determinem a causa exata da morte e que também diferencie a PIDS de outras afecções gastrointestinais dificulta a determinação da real prevalência dentro das granjas (BUDDLE \& TWOMEY, 2002; STRAW et al., 2002).

Em 1976, relatou-se mortalidade de 28 suínos ao ano, entre animais de terminação e machos adultos, em decorrência de PIDS, em cinco rebanhos na GrãBretanha (ALLEN \& SAUNDERS, 1976). DESROSIERS (2008) relata que, em um grande sistema de integração de suínos no Brasil, foi observado que 19,3\% das mortes ocorridas entre animais de 60 a 120 dias de vida foram em decorrência de PIDS. No entanto, em animais mais velhos, essa taxa pode subir para 45,5\%. A maior parte das mortes ocorre em animais de melhor conformação corporal e, portanto, maior valor econômico agregado (STRAW et al., 2002; HOLLIS, 2006; LABUSCAGNE, 2009; SONCINI, 2010). Segundo SONCINI (2010), os prejuízos com a PIDS em muitas granjas é superior ao do descarte de animais refugos.

Existe pouca pesquisa científica a respeito de mortalidade de suínos com este quadro e faltam publicações com relatos da ocorrência de PIDS e discussão de prováveis fatores de risco envolvidos. Neste trabalho, são discutidos os principais fatores predisponentes para a ocorrência de PIDS, sua fisiopatologia e o diagnóstico adequado desta afecção.

Sinais clínicos e diagnóstico

O primeiro e principal sinal da ocorrência de PIDS em um rebanho é a morte súbita de suínos supostamente saudáveis e com boa conformação corporal, sem sinais clínicos e evolução bastante rápida (SMITH \& SHANKS, 1971; ALLEN \& SAUNDERS, 1976; BUDDLE \& TWOMEY, 2002; HOLLIS, 2006; LABUSCAGNE, 2009). Os suínos encontram-se em fase de recria e terminação, entre 70 a $120 \mathrm{~kg}$ de peso vivo, com quatro a seis meses de idade (STRAW et al., 2002), podendo ocorrer ainda em animais a partir de $45 \mathrm{~kg}$ de peso vivo (ALLEN \& SAUNDERS, 1976) ou mais jovens, a partir de dois meses (BUDDLE \& TWOMEY, 2002). Inicialmente, os animais apresentam relutância em se alimentar e se locomover, devido à dilatação do abdômen, mostram-se inquietos, ansiosos, em desconforto. Esses leitões procuram se deitar em locais frescos, tentando achar conforto, e adotam posicionamento de "cão sentado". O abdômen fica bastante tenso à palpação e eles demonstram interesse pelo alimento, apesar de ingerirem pouco ou mesmo nada (BUDDLE \& TWOMEY, 2002; THOMSON et al., 2007; MARTINEAU et al., 2008).

É difícil identificar o animal doente dentre tantos leitões dentro da baia e suspeitar deste diagnóstico, pois os sinais são bastante discretos no início do quadro. Além disso, muitos animais se recuperam, o que dificulta a estimativa de morbidade (STRAW et al., 2002), ou o quadro avança e culmina em morte durante a madrugada, quando não há funcionários vigilantes (LABUSCAGNE, 2009). Eventualmente, o quadro avança e o leitão rola no chão da baia na tentativa de minimizar o desconforto, vocaliza, possivelmente devido à dor, sendo já notável a dilatação intensa do abdômen e severa dispnéia (BUDDLE \& TWOMEY, 2002; LABUSCAGNE, 2009). A morte ocorre em aproximadamente duas horas por incapacidade respiratória devido ao aumento da pressão intra-abdominal e consequente asfixia. Devido à rapidez da evolução clínica, do grande número de animais por baia e do horário em que ocorre, essa sintomatologia normalmente passa despercebida, resultando no diagnóstico de morte súbita (BUDDLE \& TWOMEY, 2002; THOMSON et al., 2007; MARTINEAU, 2008a; MARTINEAU et al., 2008; SONCINI, 2010).

À necropsia, estes animais apresentam moderada a acentuada palidez de musculatura e vísceras e intensa dilatação abdominal (SMITH \& SHANKS, 1971; HOLLIS, 2006; THOMSON et al., 2007; 
MARTINEAU, 2008a; LABUSCAGNE, 2009). À abertura da cavidade, as alças intestinais se lançam para fora pelo excesso de pressão e observam-se alças intensamente avermelhadas, repletas por grande quantidade de gás e contendo líquido sanguinolento. A topografia das alças deve ser observada atentamente, pois pode ocorrer torção intestinal prévia, durante a evolução do quadro, ou mesmo durante a agonia de morte, quando o animal se debate (SMITH \& SHANKS, 1971; BUDDLE \& TWOMEY, 2002; STRAW et al., 2002; HOLLIS, 2006; THOMSON et al., 2007; MARTINEAU et al., 2008; LABUSCAGNE, 2009). Nesse sentido, a torção deve ser considerada um achado adicional e não ser utilizada como parâmetro diagnóstico. $\mathrm{Na}$ verdade, não há um consenso se a torção intestinal é uma das causas do quadro ou somente uma consequência (BUDDLE \& TWOMEY, 2002). O fatoé que a torção pode estar presente ou não nos casos de PIDS, sendo esta última uma designação mais abrangente e adequada do processo patológico (SMITH $\&$ SHANKS, 1971; MARTINEAU et al., 2008a).

O intestino delgado aparece frequentemente envolvido, porém somente jejuno e íleo, pois o duodeno, assim como o estômago, não recebe suprimento sanguíneo dos vasos mesentéricos e, portanto, é menos acometido quando comparado aos outros segmentos (SMITH \& SHANKS, 1971; ALLEN \& SAUNDERS, 1976; MARTINEAU, 2008a; LABUSCAGNE, 2009). A serosa das alças fica bastante avermelhada devido à acentuada congestão, porém nenhuma ou pouca alteração é observada na mucosa. O conteúdo é pastoso e contém sangue não coagulado. $\mathrm{O}$ intestino grosso apresenta lesões congestivas semelhantes. As paredes do ceco e cólon ficam intensamente avermelhadas, porém o conteúdo intestinal não é sanguinolento. Os linfonodos mesentéricos ficam bastante congestos e edemaciados e as demais vísceras abdominais apresentam-se pálidas. Geralmente, o estômago encontra-se repleto (SMITH \& SHANKS, 1971; ALLEN \& SAUNDERS, 1976; BUDDLE \& TWOMEY, 2002; STRAW et al., 2002; HOLLIS, 2006; THOMSON et al., 2007; MARTINEAU et al., 2008; LABUSCAGNE, 2009).

Ao exame histopatológico, não se observam alterações estruturais ou inflamatórias, possivelmente devido ao curto tempo de evolução do quadro. Nos segmentos de intestino delgado e grosso, observamse somente alterações circulatórias severas como engurgitamento de vasos, hemorragia na lâmina própria e submucosa e extravasamento de hemácias na superfície de vilosidades, por diapedese (SMITH \& SHANKS, 1971; STRAW et al., 2002; THOMSON et al., 2007). É bastante importante realizar diagnóstico diferencial neste tipo de quadro, pois a presença de sangue no lúmen intestinal pode levar a outras suspeitas clínicas, como enteropatia proliferativa dos suínos, causada pela bactéria Lawsonia intracellularis (BUDDLE \& TWOMEY, 2002; LABUSCAGNE, 2009), salmonelose, disenteria suína (STRAW et al., 2002; HOLLIS, 2006), entre outras. Nesses casos, a parede intestinal afetada está marcadamente espessada pela inflamação, pode-se observar necrose da superfície da mucosa e presença de sangue coagulado ou excesso de muco no lúmen intestinal. O diagnóstico histológico édeterminante (GUEDES, ROBERTO, 2010, informação pessoal).

Fatores predisponentes e fisiopatologia

A rápida evolução da suinocultura seguramente contribuiu para o aumento da ocorrência da PIDS. A grande evolução nas áreas da genética e nutrição ao longo dos anos exige grande esforço fisiológico por parte dos animais para rápido crescimento e ganho de peso. $\mathrm{O}$ aumento da dimensão das granjas, com alojamento de muitos animais em baias de terminação, mão de obra restrita, equipamentos que não comportam a evolução da produção, arraçoamento com restrição alimentar, deficiência de espaço nos comedouros das baias, que geram competitividade entre os animais e estresse constante no momento da alimentação são algumas das condições predisponentes à morte súbita por PIDS (HOLLIS, 2006; LABUSCAGNE, 2009; SONCINI, 2010).

A PIDS acomete animais saudáveis em fase de crescimento e terminação, mas pode também afetar adultos. Sugere-se que a alimentação e os hábitos alimentares sejam fatores de risco (BUDDLE \& TWOMEY, 2002; HOLLIS, 2006; MARTINEAU, 2008a; LABUSCAGNE, 2009). Leitões jovens comem pequena quantidade de alimento diversas vezes ao dia, enquanto leitões mais velhos e mais pesados ingerem quantidade maior de alimento por refeição e menos vezes ao dia (STRAW et al., 2002; HOLLIS, 2006; MARTINEAU, 2008b; LABUSCAGNE, 2009). Por motivos ainda indeterminados, há excessiva produção de gases nas alças intestinais. Consequentemente, ocorre intenso aumento da pressão intrabdominal, que leva à congestão de vasos mesentéricos por hiperemia passiva local aguda. Instala-se um quadro de choque hipovolêmico, ocorre um desbalanço ácido-básico grave e morte (BUDDLE \& TWOMEY, 2002; THOMSON et al., 2007; MARTINEAU, 2008b; MARTINEAU et al., 2008; LABUSCAGNE, 2009). Estômago e intestino toleram hipoperfusão sanguínea por um período curto de tempo, observando-se alterações circulatórias e hemodinâmicas graves 
quando a dilatação abdominal persiste por um tempo prolongado (GUDMUNDSSON, 2001).

O manejo de arraçoamento entre granjas é variável podendo ser ad libitum ou de restrição, com trato oferecido duas ou três vezes ao dia. A alimentação em grande quantidade, poucas vezes ao dia, contraria a fisiologia normal do trato digestivo dos suínos e, assim, sujeita as alças intestinais à distensão e rotação por estarem muito repletas. Deveriam, portanto, realizar pequenas e constantes refeições ao longo do dia (STRAW et al., 2002; HOLLIS, 2006; MARTINEAU, 2008a). O fornecimento de ração úmida, fabricada com veículo aquoso ou com substrato proveniente de subprodutos do leite, também está relacionado à ocorrência de PIDS, porém há relatos de ocorrência em rebanhos em que se oferecem somente rações secas (BUDDLE \& TWOMEY, 2002; THOMSON et al., 2007; SONCINI, 2010). Outro fator que parece contribuir é a agitação dos animais antes de receberem o alimento, como em brincadeiras ou brigas entre leitões, remanejamento de lotes, ou mesmo o exercício forçado após alimentarem-se, associado à ingestão de quantidade exagerada de alimento (STRAW et al., 2002; HOLLIS, 2006; MARTINEAU, 2008a; LABUSCAGNE, 2009; SONCINI, 2010).

A utilização de soro de leite, fresco ou fermentado, para complementar a dieta por sua natureza rica em proteínas, ou o fornecimento de rações com substrato fermentável foram diretamente relacionadas à ocorrência da PIDS e, recentemente, a possível patogenia foi proposta (BUDDLE \& TWOMEY, 2002; THOMSON et al., 2007). Sugere-se que a dilatação abdominal é causada por uma excessiva produção de gases provenientes da fermentação da lactose. THOMSON et al. (2007) justificam que, após o desmame, a mucosa intestinal sofre modificações e diminui a disponibilidade de lactase ao longo do tempo e se esgota completamente em animais adultos. Assim, fica prejudicada a digestão da lactose proveniente do soro, que não pode ser hidrolisada e transformada em glicose e galactose. Essa molécula fica disponível à fermentação nas alças intestinais, com consequente produção de ácido lático, gás metano e hidrogênio. Ainda, ocorre desequilíbrio de microbiota, favorecendo a proliferação de bactérias anaeróbias também produtoras de gases (BUDDLE \& TWOMEY, 2002; LABUSCAGNE, 2009).

Existe predisposição anatômica para a ocorrência de PIDS em suínos, uma vez que os vasos mesentéricos craniais estão localizados adjacentes ao duodeno e ao cólon transverso e a cavidade abdominal é um ambiente com espaço limitado para expansão das vísceras (MARTINEAU, 2008b). A intensa dilatação das alças do cólon comprime a veia mesentérica e desencadeia uma cascata de eventos irreversíveis. A compressão venosa leva à congestão, hipertensão portal, estagnação sanguínea, extravasamento de sangue dos capilares para tecidos adjacentes e lúmen intestinal e, consequentemente, hipóxia. Ocorre um aumento na taxa de catabolismo celular e o animal entra em acidose metabólica. Há vasodilatação com diminuição da resistência vascular periférica e instalase um quadro de choque hipovolêmico (Figura 1) (STRAW et al., 2002; THOMSON et al., 2007; MARTINEAU, 2008b; MARTINEAU et al., 2008).

Quando é possível aferir a pressão intragástrica através de um tubo esofágico acoplado a um manômetro, observam-se sinais de desconforto quando a pressão interna atinge valores superiores a $30 \mathrm{mmHg}$, pois interfere na pressão da veia porta. A pressão arteriovenosa cai notavelmente, justificando a diminuição da perfusão sanguínea local. Com isso, ocorre vasoconstrição por secreção de fatores vasoativos e consequente aumento da resistência vascular periférica nos vasos do intestino delgado. A pressão intrabdominal pode atingir $40 \mathrm{mmHg}$, ou seja, dez vezes superior à pressão normal, e após três horas de exposição contínua à 30mmHg, já é possível observar alterações hemodinâmicas consideráveis que oferecem risco à vida do animal (GUDMUNDSSON et al., 2001). Há diminuição de aporte sanguíneo à veia porta, por aumento da resistência. Essa crescente pressão intrabdominal dificulta a contração do diafragma e da musculatura intercostal, impossibilita a respiração e leva à morte do leitão por asfixia (GUDMUNDSSON \& HELTNE, 2004; THOMSON et al., 2007; MARTINEAU, 2008b; MARTINEAU et al., 2008).

Em geral, as vísceras mantêm a topografia anatômica, mas a torção das alças sobre um ponto fixo pode ocorrer. Não é um evento obrigatório dentro desse quadro, podendo ou não estar presente, como mencionado anteriormente (SMITH \& SHANKS, 1971; BUDDLE \& TWOMEY, 2002; MARTINEAU, 2008a). A diferença de pressão em diferentes segmentos pode levar à torção parcial ou completa das alças intestinais, que se deslocam a fim de uma melhor acomodação dentro da cavidade (THOMSON et al., 2007; MARTINEAU, 2008b; MARTINEAU et al., 2008). A intensa dilatação dos segmentos intestinais leva à expansão do comprimento da curvatura maior da alça, uma vez que a face mesentérica do intestino tem capacidade inferior de dilatação, quando comparada à face anti-mesentérica (BUDDLE \& TWOMEY, 2002).

O envolvimento bacteriano na PIDS é bastante controverso. Especula-se a respeito da participação do Clostridium perfringens, assim como 


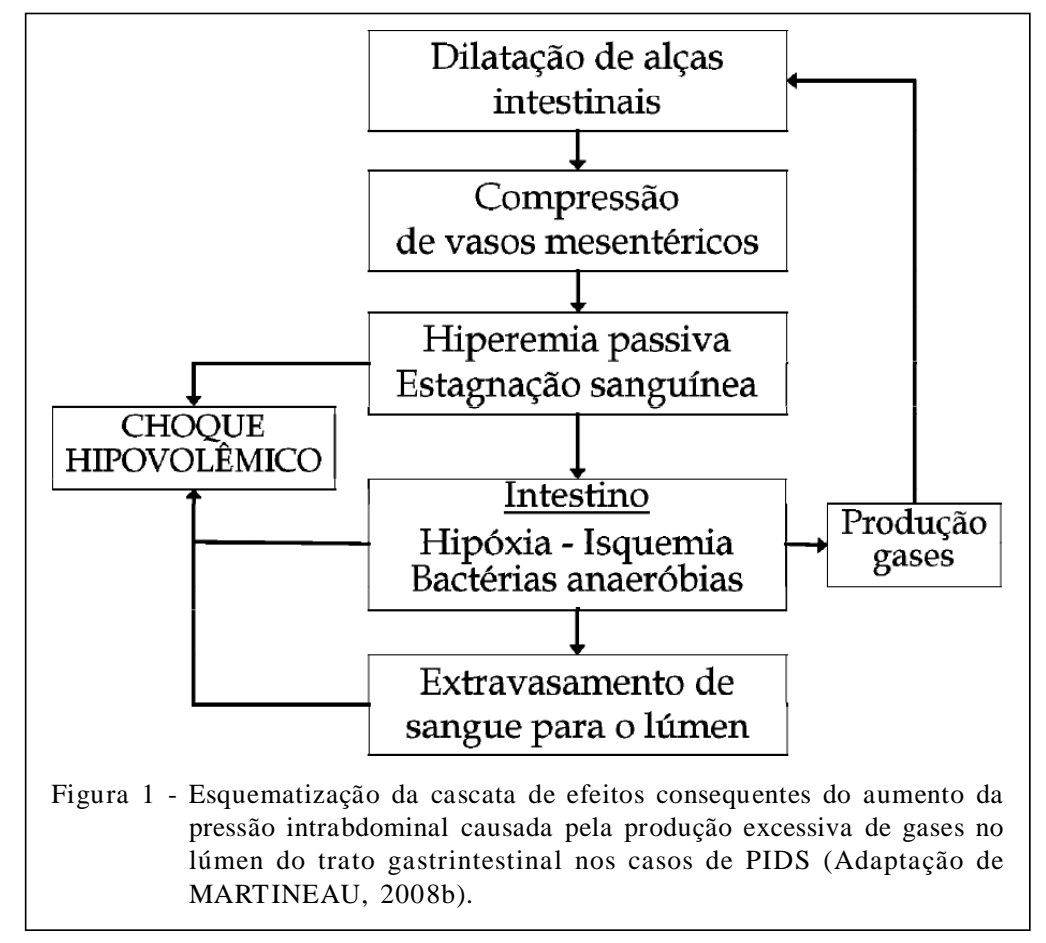

da Escherichia coli, porém nada foi comprovado (SMITH \& SHANKS, 1971; BUDDLE \& TWOMEY, 2002; STRAW et al., 2002; THOMSON et al., 2007; LABUSCAGNE, 2009). Essa hipótese se baseia no isolamento desses agentes de animais que morrem com a síndrome, assim como na redução do número de casos com o uso preventivo de framomicina (SMITH \& SHANKS, 1971), clortetraciclina e bacitracina na ração (STRAW et al., 2002). Importante ressaltar que tanto Clostridium sp quanto Escherichia coli fazem parte da microbiota intestinal e, consequentemente, isolados de animais sadios ou com causa morte não relacionada a PIDS.

A intolerância a componentes do soro de leite, como a caseína, também é citada como condição predisponente, mas não se aplica logicamente aos casos de PIDS em animais que não recebem subprodutos do leite na dieta (MARTINEAU, 2008a). A estação do ano pode ser um fator relevante, uma vez que, em períodos muito quentes, os animais ficam desconfortáveis, o que gera maior estresse dentro das baias (ALLEN \& SAUNDERS, 1976; STRAW et al., 2002; HOLLIS, 2006; THOMSON et al., 2007; LABUSCAGNE, 2009). Além disso, supostamente, os animais não se alimentariam durante o dia devido ao calor e sim à noite, quando o clima está mais fresco, justificando a observação frequente de morte pela manhã de animais previamente sadios (STRAW et al., 2002; THOMSON et al., 2007; LABUSCAGNE, 2009). STRAW et al. (2002) sugerem maior incidência em machos e justificam que a castração e as variações hormonais a que esses animais são submetidos poderia influenciar no diagnóstico.

Prevenção

A PIDS é considerada multifatorial, porém etiologia e patogênese não são bem definidas, o que dificulta seu controle. Prevenção envolve redução de fatores de risco. Visto que o fornecimento de soro de leite é um fator agravante, deve-se restringi-lo. Uma opção seria fornecê-lo após 24 a 48 horas de estocagem, quando o produto é fermentado por lactobacilos e produz ácido lático previamente, evitando a produção gasosa no interior das alças intestinais (THOMSON et al., 2007; MARTINEAU, 2008b). Ainda, restringir a ingestão de soro de leite a $20 \%$ de ração seca, preferir rações secas às úmidas, oferecer rações peletizadas uma hora antes de fornecer alimentos muito fermentáveis, como o soro, aumentar a quantidade de fibras da ração, fornecer alimento aos leitões mais vezes ao dia ou até mesmo ad libitum (BUDDLE \& TWOMEY, 2002; HOLLIS, 2006; THOMSON et al., 2007; MARTINEAU et al., 2008).

A utilização de antimicrobianos preventivamente foi citada como efetiva. SMITH \& SHANKS (1971) e STRAW et al. (2002) relatam que a administração de ração medicada com antimicrobianos diminui sensivelmente a ocorrência de PIDS, pois, apesar de não ser comprovada etiologia bacteriana, a 
droga auxilia no controle da proliferação de bactérias produtoras de gás existentes na microbiota intestinal, porém é um procedimento de alto custo, que eleva o custo de produção e possui embargo em diversos países no mundo, o que inviabiliza esta opção (THOMSON et al., 2007).

São várias as medidas paliativas utilizadas no controle da ocorrência de PIDS, em especial estão as relacionadas à higiene na produção de ração, das baias e comedouros e garantia de qualidade da água fornecida aos animais (SONCINI, 2010). A redução do estresse, em especial o relacionado à densidade animal, mantendo-se $0,7 \mathrm{~m}^{2}$ por suíno alojado, é recomendada. Ainda, indica-se a melhora no arejamento das baias com sistemas de ventilação eficientes, particularmente no verão, evitando-se o super aquecimento e o acúmulo de gases (STRAW et al., 2002; HOLLIS, 2006). $\mathrm{O}$ oferecimento de quantidade e área suficientes de comedouros para o número de animais alojados é uma das ações com melhores resultados, associado ao aumento de arraçoamentos por dia, melhora na qualidade da mão-de-obra para a dimensão da granja e proporção de animais alojados. Manejo adequado deve minimizar as situações de estresse, como evitar misturas excessivas de lotes para não predispor os animais às situações de hierarquia e, assim, os leitões não deixam de se alimentar para entrarem em brigas, nem irãoingerir grandes quantidades de alimento rapidamente por competição (HOLLIS, 2006; LABUSCAGNE, 2009; SONCINI, 2010).

\section{CONCLUSÃO}

A PIDS é uma patologia de causa multifatorial, ao que tudo indica não infecciosa e sim alimentar, com envolvimento de fatores ambientais. Essa condição vem crescendo em importância com o aumento de sua ocorrência, ou por ter sido mais relatada nos últimos anos. É uma condição subdiagnosticada que merece maior atenção por parte de produtores e médicos veterinários devido ao significativo impacto econômico causado pela mortalidade de animais de considerável valor agregado. A identificação de fatores de risco em cada granja com implementação de melhorias para minimizá-los e investimentos em melhor treinamento de pessoal envolvido com a suinocultura é fundamental para seu efetivo controle.

\section{REFERÊNCIAS}

ALLEN, M.M.; SAUNDERS, R.W. The intestinal haemorrhage syndrome in centrally tested pigs in great Britain. In: INTERNATIONAL PIG VETERINARY SOCIETY CONGRESS, 4., 1976, Ames, USA. Proceedings... Ames, Iowa, IPVS, 1976. p.5.
BUDDLE, J.R.; TWOMEY, D.E. The "porcine intestinal distension syndrome". Pig Journal, v.50, p.68-82, 2002. Disponível em: <http://www.thepigsite.com /pigjournal/volume/ 50>. Acesso em: 01 set. 2010.

DESROSIERS, R. More on intestinal torsions/HBS/PIDS Part 2. International Pigletter, v.28, n.9b, p.1-2, 2008.

GUDMUNDSSON, F.F. et al. Effects of prolongated increased intra-abdominal pressure on gastrointestinal blood flow in pigs. Surgical Endoscopy, v.15, p.854-860, 2001. Disponível em: <http://www.springerlink.com/content/4yq10t $2 \mathrm{mt} 9 \mathrm{kwrdmk} /$ fulltext.pdf $>$. Acesso em: 20 out. 2010. doi: 10.1007/ s004640090090.

GUDMUNDSSON, F.F.; HELTNE, J.K. Respiratory changes during prolonged increased intra-abdominal pressure in pigs. Acta Anaesthesiologica Scandinavica, v.48, p.463-468, 2004. Disponível em: <http://onlinelibrary.wiley.com/doi/ 10.1111/j.0001-5172.2004.0354.x/pdf >. Acesso em: 20 out. 2010. doi: 10.1111/j.1399-6576.2004.00354.x.

HOLLIS, W.L. Will nutrition and management stop hemorrhagic bowel syndrome? A veterinarian's perspective. In: SWINE NUTRITION CONFERENCE, 2006, Indiana, USA. Proceedings... Indianapolis: Midwest Swine Nutrition, 2006. p.38-41. Disponível em: <http://www.livestocktrail.uiuc.edu/ uploads/porknet/papers/MWSNC\%20Proceedings-2006.pdf>. Online. Acesso em: 17 fev. 2011.

LABUSCAGNE, A. Haemorrhagic bowel syndrome in grower pigs. 2009. 92f. Monografia (obtenção de título de especialista) - Department of production animal studies, University of Pretoria. Disponível em: <http://upetd.up.ac.za/ thesis/available/etd-0 $8132010-143946 /$ unrestricted/ dissertation.pdf>. Acesso em: 21 fev. 2011.

MARTINEAU, G. The BB dead pig: Part 1. International Pigletter, v.27, n.12c, p.1-2, 2008a.

MARTINEAU, G. The BB dead pig: Part 2. International Pigletter, v.27, n.12b, p.1-2, 2008b.

MARTINEAU, G. et al. Le syndrome de distension intestinale porcin (SDIP) (<l'entérotoxémie >). Journées Recherche Porcine, v.40, p.33-42, 2008. Disponível em: <http:// www.journees-recherche-porcine.com/texte/2008/sante/ s06.pdf>. Acesso em: 20 out. 2010.

SMITH, W.J.; SHANKS, P.L. Intestinal haemorrhage syndrome. Veterinary Record, v.89, p.55-56, 1971.

SONCINI, R.S. Torção do mesentério. Porkworld. v.58, ano 10, p.156-159, 2010.

STRAW, B. et al. Factors associated with death due to hemorrhagic bowel syndrome in two large commercial swine farms. Journal of Swine Health and Production, v.10, n.2, p.75-79, 2002. Disponível em: <http://www.aasv.org/shap/ issues/v10n2/v10n2p75.pdf $>$. Acesso em: 20 out. 2010.

THOMSON, J.R. et al. Pressure-related abdominal changes in pigs with 'whey bloat' - a case report. Pig Journal, v.59, p.152-159, 2007. Disponível em: <http://www.thepigsite.com/ pigjournal/volume/59>. Acesso em: 20 out. 2010. 